\title{
MAPAS CONCEPTUALES COMO INSTRUMENTO DE COORDINACIÓN DOCENTE EN ESTUDIOS DE POSGRADO
}

\author{
J. Vicente García-Tormo \\ Facultad de Ciencias de la Actividad Física y del Deporte \\ Universidad de León \\ jvgart@unileon.es
}

Recepción Artículo: 26 febrero 2020

Admisión Evaluación: 4 marzo 2020

Informe Evaluador 1: 13 marzo 2020

Informe Evaluador 2: 15 Marzo 2020

Aprobación Publicación: 20 abril 2020

\section{RESUMEN}

Con el actual Espacio Europeo de Educación Superior, el trabajo en equipo y la coordinación son estrategias esenciales para mejorar la calidad de la enseñanza, implicando tomar conciencia de la importancia de entender la docencia como una responsabilidad compartida en la que participarán tanto docentes como estudiantes. Para ello es importante establecer mecanismos y recursos para procurar una óptima coordinación (intra-materia e inter-materias). La Coordinación Académica, entre otras tareas, persigue que no existan solapamientos de contenidos entre las distintas asignaturas, garantizando que se cubra el abanico de competencias propias del título.

En el contexto del Máster Universitario en Entrenamiento y Rendimiento Deportivo de la Universidad de León, se ha visto la necesidad de optimizar la coordinación inter e intra-asignatura, para evitar solapamiento de contenidos detectados en el programa de Plan de Acción Tutorial en el que participan los estudiantes. Con este fin, se está llevando a cabo un proyecto de innovación docente para introducir un instrumento de coordinación basado en mapas conceptuales, que permitiese de manera visual, controlar y ubicar los contenidos de cada materia.

Los responsables de cada materia han participado en una jornada de familiarización con el instrumento que se mostrará a los estudiantes en la primera sesión presencial y en cada uno de los bloques de contenidos, contextualizando tanto la materia como los contenidos.

Este instrumento será valorado tanto por el profesorado como por los estudiantes mediante un cuestionario on-line (Google forms), con el que se obtendrá un Feedback que permitirá ajustar y valorar su utilidad para cursos posteriores y en otras titulaciones.

Este trabajo se ha desarrollado dentro del Plan de apoyo a la innovación docente de la Universidad de León (PAID 2019), bajo el título "Aplicación de mapas conceptuales como instrumento de coordinación docente en estudios de posgrado".

Palabras clave: estudios de postgrado; coordinación académica; mapas conceptuales; contenidos 


\section{MAPAS CONCEPTUALES COMO INSTRUMENTO DE COORDINACIÓN DOCENTE EN ESTUDIOS DE POSGRADO}

\section{ABSTRACT}

Concept maps as an instrument of teaching coordination in postgraduate studies. In the current European Higher Education Area, teamwork and coordination are essential strategies to improve the quality of teaching, involving awareness of the importance of understanding teaching as a shared responsibility in which teachers and students alike will participate. It is important to establish mechanisms and resources to ensure optimal coordination (intra and inter-material). The Academic Coordination, among other tasks, seeks to ensure that there is no overlap in content between the different subjects, guaranteeing that the range of skills of the degree is covered.

In the context of the University Master's Degree in Training and Sports Performance of the University of León, it has been necessary to optimize the inter- and intra- subject coordination, to avoid overlapping of contents detected in the Tutorial Action Program, in which students participate. To this end, a teaching innovation project is being carried out to introduce a coordination instrument based on concept maps, which would make it possible to visually control and locate the contents of each subject.

Professors of each subject have participated in a familiarization session with the instrument that will be shown to students in the first presential session of each subject, contextualizing both the subject and the contents.

This instrument will be evaluated by the teaching staff and the students by means of an on-line questionnaire (Google forms), with which feedback will be obtained that will allow the adjustment and evaluation of its usefulness for later courses and other degrees. This study has been developed within the Plan of support for teaching innovation of the University of León (PAID 2019), under the title "Application of concept maps as an instrument of teaching coordination".

Keywords: postgraduate studies; academic coordination; concept maps; contents

\section{INTRODUCCIÓN}

Con el actual Espacio Europeo de Educación Superior, el trabajo en equipo y la coordinación son estrategias esenciales para mejorar la calidad de la enseñanza, tomando conciencia de la importancia de entender la docencia como una responsabilidad compartida en la que participarán tanto docentes como estudiantes (Antúnez, 1999; Fuentes-Guerra, Cabrera, del Mar, Llorent García, y Olivares García 2012; Fernández-Larragueta y Rodorigo, 2016). Esta coordinación va a estar presente desde el diseño del título hasta el último de los aspectos relacionados con el proceso formativo, repercutiendo en la calidad de la formación que recibirán los estudiantes (González y Asensio, 2006). Esta coordinación y la capacidad de trabajo de los docentes implicados en las asignaturas, va a condicionar el rendimiento académico y la percepción subjetiva de los estudiantes sobre el título en general y las asignaturas en particular (Lavié, 2009; Santos et al., 2009).

En el contexto de los estudios de posgrado, y en concreto en el Máster en Entrenamiento y Rendimiento de la Universidad de León (Máster ERD), esta coordinación recae directamente en el Coordinador del Título y en la Comisión Académica del título, la cual debe establecer mecanismos y recursos para procurar una óptima coordinación (intra-materia e inter-materias), lo que facilitará lograr una enseñanza de calidad (Sánchez Jiménez, y Galiano Coronil, 2019).

La Coordinación Académica (inter-materias), entre otras tareas, persigue que no existan solapamientos de contenidos entre las distintas asignaturas. Para ello se ha de supervisar las guías docentes y garantizar que se cubra el abanico de competencias propias del título. Para ello, es necesario que se coordinen los diferentes responsables de las materias, dando las directrices oportunas para establecer una homogeneidad en los aspectos relacionados con el proceso formativo.

Paralelamente, la Coordinación de Asignatura (intra-materia), persigue un óptimo desempeño entre los diferentes docentes implicados en la misma, planificando las actividades formativas y los contenidos, que se verá reflejado en una mejora de la actividad docente (Velasco, Bailón, Terrón, y García, 2012), con vistas a que los estudiantes logren adquirir las competencias específicas de su asignatura. Estas funciones recaen principalmente sobre el Profesor Responsable de cada materia. 
Esta coordinación intra-materia es de gran importancia como punto de partida para lograr una formación de calidad (Learreta Ramos, 2006). Concretamente en el Máster ERD, esta labor se ve dificultad por la participación de numerosos docentes/profesionales de reconocido prestigio ajenos a la Universidad de León, que enriquecen la formación del alumno aportando su experiencia directa en diferentes ámbitos de especialización. Esta participación del profesorado externo requiere un esfuerzo por parte de los responsables de las materias para lograr una coordinación y una coherencia entre los bloques de contenidos que se imparten.

Dada la importancia de esta coordinación y con el objetivo de mejorarla, la Comisión Académica del título ha propuesto un proyecto de innovación docente, aprovechando la información que se recoge de la participación de los estudiantes en los Planes de Acción Tutorial de la Universidad de León, en el que se identifican posibles necesidades del título, así como posibles solapamientos en los contenidos.

Esta propuesta está basada en el diseño, elaboración y uso de mapas conceptuales como recurso docente (Cabaco, y Berrocoso, 2008). Estos instrumentos ofrecen la posibilidad de recoger información de forma ordenada y esquematizada, concretamente los contenidos que se van a desarrollar en cada una de las materias. Esta estructura permite que los estudiantes tengan una representación gráfica de la materia que le facilite relacionar los contenidos entre si, evitando que solo se atienda a relaciones lineales en los procesos de aprendizaje (Barroso, 2006).

\section{OBJETIVOS}

El objetivo general de este trabajo se centra en diseñar, crear y aplicar un instrumento basado en mapas conceptuales para la coordinación inter e intra-asignatura, para su utilización estudios de posgrado.

Para lograr este objetivo principal, se fijaron otros objetivos específicos:

- Llevar cabo un proceso formativo inicial para familiarizar a los Profesores Responsables de las materias, con el diseño y uso de los mapas conceptuales para coordinar los contenidos de sus asignaturas.

- Aplicar en la práctica el instrumento propuesto en cada una de las materias del título.

- Publicar los mapas conceptuales de las diferentes materias a través de la web propia del Máster ERD, con el fin de aportar a los estudiantes (presentes y futuros) una visión de conjunto de los contenidos que incluye el título.

- Diseñar y crear un instrumento on-line de evaluación (formulario a través de Google Forms) para valorar Ios potenciales y debilidades que presentan los mapas conceptuales aplicados en el curso 2019-20.

\section{MUESTRA}

La muestra de este trabajo comprenderá la totalidad de estudiantes matriculados en las asignaturas obligatorias y optativas del Máster en Entrenamiento y Rendimiento Deportivo de la Universidad de León ( $\mathrm{n}=26$ ), aplicándose en la mayoría de las asignaturas del título.

\section{METODOLOGÍA}

Este proyecto se ha desarrollado a lo largo del presente curso 2019/2020 en las tres asignaturas obligatorias y ocho de las asignaturas optativas del Máster ERD de la Universidad de León.

Con el fin de una adecuada aplicación del instrumento propuesto, este proyecto se ha desarrollado describiendo las siguientes etapas metodológicas (Figura 1).

\section{Fase 1: Formación del profesorado.}

Los profesores responsables de las materias del Máster ERD, asistirán a una jornada de coordinación académica y familiarización con el instrumento propuesto, estableciendo los criterios de elaboración de los mapas conceptuales en función de los contenidos específicos de cada materia (Tamayo, 2006). 


\section{MAPAS CONCEPTUALES COMO INSTRUMENTO DE COORDINACIÓN DOCENTE EN ESTUDIOS DE POSGRADO}

\section{Fase 2: Elaboración de los mapas conceptuales.}

A partir de las indicaciones establecidas en la jornada de formación, los profesores responsables elaborarán el mapa conceptual de su asignatura, estableciendo una jerarquía, vinculación y/o secuencia de contenidos que plasmarán en un instrumento gráfico que se le facilitará a todos los implicados en la materia (docentes, estudiantes y personal de apoyo).

\section{Fase 3: Aplicación.}

Al comienzo de cada asignatura, en la primera sesión presencial, el profesor responsable mostrará y explicará el mapa conceptual específico de su asignatura, contextualizando cada uno de los contenidos. Esto se repetirá al comienzo de cada sesión o bloque de contenidos, con el fin de que el alumnado se ubique e identifique de forma visual en contenido formativo que se va a desarrollar a continuación.

\section{Fase 4: Análisis de los resultados.}

En la segunda sesión del Plan de Acción Tutorial que se realiza al comienzo del segundo semestre, Ios tutores solicitarán a los estudiantes que valoren la utilidad y adecuación del uso de este instrumento en las asignaturas que han cursado hasta dicho momento. Por su parte, el responsable del proyecto consultará a los profesores responsables de las asignaturas del primer semestre sobre la elaboración y uso de este instrumento. Estas valoraciones se tendrán en cuenta para realizar adaptaciones y/o mejoras en el uso del instrumento de coordinación de las asignaturas del segundo semestre.

\section{Fase 5: Revisión del proceso.}

A final de curso, se llevará a cabo una actividad de evaluación en el que participarán todos los implicados en el proceso formativo (docentes, estudiantes y personal de apoyo), los cuales valorarán el uso de este instrumento. Para ello se empleará un formulario elaborado con la herramienta Google Forms y que podrán contestar a través de sus dispositivos electrónicos (ordenador, Smartphone o Tablet). Tras esta evaluación, se valorarán posibles mejoras y su continuidad en cursos posteriores.

Figura 1: Cronología de las fases metodológicas.

\begin{tabular}{|l|l|l|l|l|l|l|l|l|l|l|l|}
\hline \multicolumn{1}{|c|}{ FASES } & Jul & Sept & Oct & Nov & Dic & Ene & Feb & Mar & Abr & May & Jun \\
\hline $\begin{array}{l}\text { 1. Formación del } \\
\text { profesorado }\end{array}$ & & & & & & & & & & \\
\hline $\begin{array}{l}\text { 2. Elaboración mapas } \\
\text { conceptuales }\end{array}$ & & & & & & & & & & \\
\hline 3. Aplicación & & & & & & & & & & \\
\hline 4. Análisis resultados & & & & & & & & & & \\
\hline 5. Revisión del proceso & & & & & & & & & & \\
\hline
\end{tabular}

\section{Herramientas utilizadas}

Para el desarrollo de este proyecto se han empleado principalmente dos herramientas:

- Para la elaboración de los mapas conceptuales (instrumento de coordinación docente) se propone el uso del software de elaboración de presentaciones más extendido en el contexto de la enseñanza como es el PowerPoint de Microsoft, el cual está disponible dentro del ámbito de acuerdo EES (Enrollment for Education Solutions) que ofrece a todo el PDI y PAS de la Universidad de León. Con esta herramienta, los responsables de las asignaturas, y en base a un modelo que se facilita desde la coordinación del título y del proyecto, podrán elaborar el mapa conceptual de cada una de sus materias, de forma sencilla e intuitiva, quedando un instrumento visual de fácil comprensión.

- Para llevar a cabo la evaluación del instrumento de coordinación docente propuesto, se diseñará un formulario con el que recoger el grado de satisfacción, las opiniones y propuesta de mejora. Para ello se recurrirá a 
una de las aplicaciones de la compañía Google, con la que actualmente la Universidad de León dispone de un acuerdo corporativo, los denominados formularios Google (Google Forms) integrados dentro de la herramienta Google Drive, ya empleado en proyectos en el contexto del Máster ERD de la Universidad de León (García-Tormo, 2018). Esta herramienta permite elaborar formularios/cuestionarios de forma intuitiva, rápida y sencilla para la recogida de información on-line, accesible para todos los miembros de la comunidad universitaria que dispongan de una cuenta de correo institucional. Esta aplicación facilitará la recogida de datos de forma inmediata y centralizando los resultados para su posterior análisis.

\section{Procedimiento}

Tras ser analizada la información recogida en las sesiones del Plan de Acción Tutorial de la Universidad de León, se detectó en el Máter ERD que existía un cierto solapamiento de contenidos entre las asignaturas del título. Este hecho motivó a la Comisión Académica de la titulación a proponer un proyecto de innovación docente para identificar y evitar los posibles solapamientos de contenidos inter e intra-asignatura. La propuesta se desarrolló en torno al uso de los mapas conceptuales, como instrumento gráfico, en los que recoger los contenidos propios de cada materia.

En una primera fase, el profesorado responsable de las materias del título, recibieron una formación con la que se familiarizaron con el instrumento propuesto, estableciéndose los criterios de elaboración en función de los contenidos específicos de cada materia.

Una vez realizada la formación, los estudiantes fueron informados de que iban a tener a su disposición este instrumento de cada una de las materias del título, el cual les serviría de guía y contextualización de los contenidos que iba a recibir en las asignaturas. Estos mapas conceptuales se les facilitaría al comienzo de cada asignatura y también los tendrían a su disposición en la página web del Máster (www.master-erd.info).

Los mapas conceptuales son elaborados por parte del profesor responsable de cada asignatura, recogiendo y ordenando los contenidos específicos de su materia, facilitando una visión de conjunto de todos los bloques de contenidos que se tratarían en el desarrollo de la asignatura. En la primera sesión presencial de cada asignatura, el profesor responsable les facilitaría a los estudiantes el mapa conceptual, el cual será usado durante el desarrollo de la asignatura, principalmente al comenzar un nuevo contenido.

A mitad de curso, y aprovechando la segunda sesión del Plan de Acción Tutorial, se solicitaría a los estudiantes que valorasen la utilidad de los mapas conceptuales como instrumento de coordinación y contextualización de los contenidos de las asignaturas. De igual modo, el profesorado implicado en las asignaturas del primer semestre también sería tenido en cuenta para valorar este instrumento. Con esta información se procedería a mejorar el instrumento de cara a las asignaturas de final de curso.

Al finalizar el curso, tanto los estudiantes como el profesorado, recibirán un cuestionario de evaluación final que sería cumplimentado por los implicados (estudiantes y profesorado) de forma telemática, enviándoles por parte del responsable del proyecto un enlace (link) que permitía a cada uno responder a las preguntas del formulario con máxima flexibilidad y libertad, eligiendo el momento y dispositivo utilizado.

Una vez se tengan los resultados de los cuestionarios, se llevará a cabo una reunión académica en la que participarán todos los profesores responsables, representantes de estudiantes y personal de apoyo implicado, con el fin de valorar el instrumento, proponer mejoras y decidir si se continua con el uso de los mapas conceptuales de cara al próximo curso académico en función del feedback y la experiencia obtenida.

\section{Resultados}

En el momento de presentar este trabajo, el proyecto se encuentra en desarrollo, pero con la puesta en práctica de este proyecto de innovación docente se esperan alcanzar los siguientes beneficios:

- El uso de los mapas conceptuales como instrumento de coordinación docente dentro de las asignaturas del Máster ERD, permitirá a todos los implicados conocer en qué momento concreto se encuentran dentro del proceso formativo, evidenciando de forma gráfica e intuitiva la jerarquía y distribución de los conocimientos dentro de la materia que se está desarrollando. 


\section{MAPAS CONCEPTUALES COMO INSTRUMENTO DE COORDINACIÓN DOCENTE EN ESTUDIOS DE POSGRADO}

- En relación al estudiante, los mapas conceptuales le permitirán tomar conciencia del desarrollo de cada asignatura de forma visual, contextualizando los contenidos que va a recibir, permitiéndole relacionar los diferentes bloques dentro de la materia y su vinculación con contenidos de otras asignaturas.

- El profesorado podrá desarrollar un instrumento propio de cada materia para coordinar a los docentes implicados en la misma y para realizar una distribución coherente de los contenidos. Esto permitirá al profesorado introducir, en cada sesión, los diferentes bloques de contenidos de una forma más clara de cara a relacionarlos con otros contenidos dentro de la materia.

- El desarrollo de este proyecto servirá de experiencia previa para la implantación del uso de este tipo de instrumentos (mapas conceptuales) en otras titulaciones de la Facultad como es el caso del Grado en Ciencias de la Actividad Física y del Deporte.

- La integración de instrumentos sencillos y visuales (mapas conceptuales), aportará al alumnado una imagen muy positiva de innovación y actualidad de la metodología empleada en este título oficial.

Parte de los resultados obtenidos en este proyecto son los mapas conceptuales que se han elaborado para cada materia (Figura 2), los cuales están disponibles en la web del Máster (www.master-erd.info).

Figura 2: Ejemplo de mapa conceptual de una asignatura obligatoria.

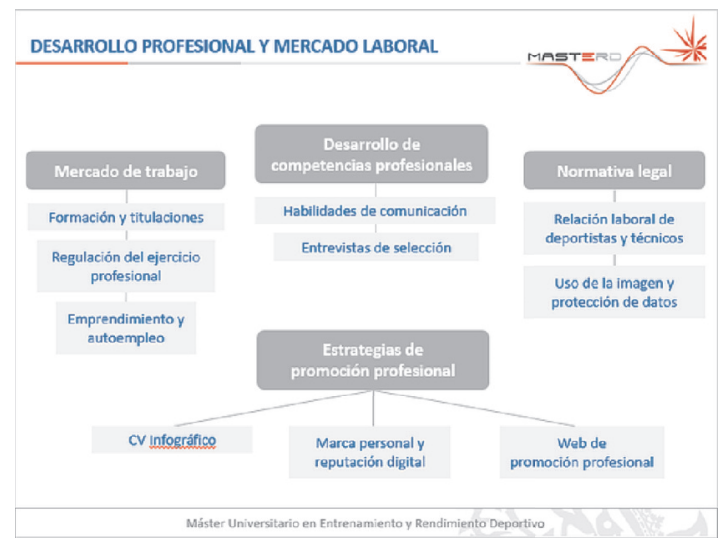

Aunque no se tienen resultados definitivo, en la primera fase de evaluación, en la que participaron estudiantes y profesores de las asignaturas del primer semestre, la valoración fue muy positiva. El feedback recibido reforzaba la utilidad de los mapas conceptuales como instrumento de coordinación.

Los estudiantes destacaron la posibilidad de ver de forma gráfica los contenidos de las asignaturas, conociendo al comienzo de la misma los bloques en los que estaba distribuidos, entendiendo las relaciones existentes entre los contenidos y posibles relaciones con otras asignaturas.

Desde el punto de vista de los docentes, se ha destacado la utilidad a la hora de distribuir y coordinar los contenidos, empleándose en la primera sesión de la asignatura y al comienzo de cada uno de los bloques de contenidos. A su vez, esta herramienta ha facilitado la contextualización de los contenidos para los docentes visitantes, conociendo que otros contenidos se desarrollan en la asignatura.

\section{CONCLUSIONES}

Aunque el proyecto no ha finalizado, a partir de los objetivos planteados inicialmente y la experiencia vivida hasta el momento, se pueden extraer las siguientes conclusiones.

Es fundamental que haya participación conjunta de estudiantes y profesorado en la mejora de un título, evidenciándose la necesidad de lograr una buena coordinación tanto entre las materias como entre el profesorado dentro de una misma asignatura. 
La propuesta de usar mapas conceptuales de las materias, ha permitido al profesorado distribuir y organizar los contenidos que se van a desarrollar, aportando una estructura que facilita el desarrollo de las asignaturas en las que participan varios docentes, principalmente cuando son externos a la Universidad de León.

Los mapas conceptuales han facilitado que los estudiantes tengan una visión general y gráfica de las asignaturas. Esto facilita la mejora del proceso de aprendizaje, ya que se contextualizan los contenidos dentro de una misma asignatura y permite relacionarlos con los de otras materias.

En general, el uso de los mapas conceptuales está aportando a la comisión académica del título un instrumento para mejorar la coordinación académica, que permite detectar posibles solapamientos de contenidos y la distribución de los mismos a lo largo del curso académico.

Este trabajo se ha desarrollado dentro del Plan de apoyo a la Innovación Docente de la Universidad de León (PAID 2019), bajo el título "Aplicación de mapas conceptuales como instrumento de coordinación docente en estudios de posgrado".

\section{REFERENCIAS BIBLIOGRÁFICAS}

Antúnez, S. (1999). El trabajo en equipo de los profesores y profesoras: factor de calidad, necesidad y problema. El papel de los directivos escolares. Educar, 24, 89-110.

Barroso, C. (2006). Planificación de la Enseñanza a través de Mapas Conceptuales. Comunidad. Universidad de la Laguna.

Cabaco, A. S., \& Berrocoso, J. V. (2008). Innovación docente e instruccional en el EEES: el mapa conceptual como estrategia facilitadora del proceso enseñanza-aprendizaje. Repensar y construir el Espacio Europeo de Educación Superior, 285-305.

Fuentes-Guerra Soldevilla, M., Cabrera, G., del Mar, M., Llorent García, V. J., \& Olivares García, M. Á. (2012). La coordinación docente universitaria desde la percepción del alumnado. Revista de Docencia Universitaria Vol.10 (2), 395-409

García-Tormo, J.V. (2018). Aplicación de TICS (formularios on-line) como metodología docente activa en Estudios de Postgrado. International Journal of Developmental and Educational Psychology, 3(1), 199-207.

González, A., \& Asensio, A. (2006). La actuación coordinada del profesorado universitario. B. Learreta (sf). La coordinación del profesorado ante las demandas del Espacio Europeo de Educación Superior, 29-35.

Larragueta, S. F., \& Rodorigo, M. (2016). Repensar la docencia universitaria: La coordinación como estrategia para la innovación. Opción: Revista de Ciencias Humanas y Sociales, (11), 514-530.

Lavié Martínez, J. M. (2009). El trabajo colaborativo del profesorado. Sevilla: Comunicación Social Ediciones y Publicaciones.

Learreta Ramos, M. B., Montil Jiménez, M. M., González Álvarez, A., \& Asensio Perales, A. (2009). Percepción del alumnado ante el uso de metodologías activas de enseñanza como respuesta a las demandas del espacio europeo de educación superior: un estudio de caso. Apunts, Educación Física y Deportes. 1ํTrimestre, 9298.

Sánchez Jiménez, M. Á., \& Galiano Coronil, A. (2019). Desarrollo de un equipo docente en la coordinación del profesorado en el ámbito universitario. ALTERIDAD. Revista de Educación, 14(1), 98-108.

Santos Rego, M. A., Lorenzo Modelo, M.M., \& Priegue Caamaño, D. (2009). Aprendizaje coopera- tivo: prácticapedagógica para el desarrollo escolar y cultural. Magis, Revista Internacional de Investigación en Educación, 2, 289-303.

Tamayo, M. F. A. (2006). El mapa conceptual una herramienta para aprender y enseñar. Plasticidad y restauración neurológica, 5(1), 62-72.

Velasco, P. J., Bailón, R. R., Terrón, M. J., \& Garcia, M. J. (2012). La coordinación del profesorado universitario: un elemento clave para la evaluación por competencias. REDU: Revista de Docencia Universitaria, 10(3), 265. 
